\title{
A second order time-varying parameters extended state observer based on hyperbolic sine function for uncertain nonlinear disturbances
}

\author{
Hongguo $\mathrm{Yu}^{1,2, \mathrm{a}}$, Zhongjian Kang ${ }^{1, \mathrm{~b}}$ and Yao Chen ${ }^{1, \mathrm{c}}$ \\ ${ }^{1}$ College of Information and Control Engineering, China University of Petroleum, Qingdao Shandong \\ 266580, China; \\ ${ }^{2}$ Research Center Automatic Control, Binzhou University, Binzhou Shandong 256603, China.

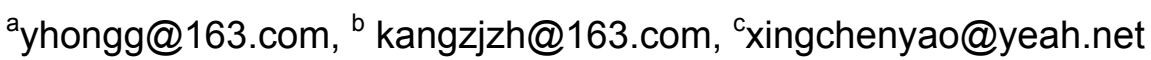

Keywords: hyperbolic sine function; extended state observer (ESO); Lyapunov function; time-varying parameters; derivative peaking.

\begin{abstract}
Derivative peaking phenomena appears in the initial stage when the initial value error between the classical extended state observer (ESO) and system state variables is bigger. A general form of extended state observer with nonlinear hyperbolic sine function is provided. The stability of a second-order ESO error system is proved with the Lyapunov function. The ESO with time-varying parameters which based on the saturation characteristic of hyperbolic tangent function was designed to realize the effectiveness of derivative peaking phenomena. Finally, the simulation experiments compared with the classical extended state observer shows that the proposed extended state observer can effectively inhibit derivative peak, and get the accurate estimation both of system state variables and uncertain nonlinear disturbances.
\end{abstract}

\section{Introduction}

The ADRC (Active Disturbance Rejection Control) technology has been widely used at home and abroad since it is proposed for many years. In recent years, ADRC technology has been extensively applied and researched on the basic theory as a kind of effective method to solve the problem of uncertain nonlinear system control ${ }^{[1]}$. The extended state observer (ESO) is the central part of the ADRC technology, which can realize the online estimation of feedback and disturbance quantity .The initial ESO is presented with nonlinear function in Literature [2], and the constructed criteria of nonlinear function is given. The classical ESO which is constructed by Fal $(\bullet)$ and Fhan (•) function were discussed systematically. A high gain linear ESO is designed in Literature [3], by choosing the sufficiently large gain parameter to ensure the estimation accuracy. A three order discrete linear ESO structure is presented and its stability analysis is given in Literature[4] . Literature [5] analyses and estimates the error of second order ESO by using non continuous piecewise Lyapunov function, and proves the stability of the system with multiple Lyapunov functions method. Literature [6] presents two improving ESO plan based on arctangent nonlinear function to obtain better control effect for the control object with output measurement noise. A kind of ADRC controller tuning method based on the controlled system time scale is presented in Literature [7], which is under the assumption of invariant observation loop time scale in ESO. Literature [8] proposes a finite time linear ESO to ensure the observation error bounded in finite time, and to realize the quick and accurate observation of the unknown disturbance and state in system. It also gives analytic relations of the observer parameters, convergence speed of the observation error and observation error of steady-state. The applications of ESO-based control strategies in diverse fields have discussed in literature [9-10].Literature [11] utilizes high gain to cancel internal and external interference for uncertain nonlinear systems. The error convergence is proved and differential peak value of initial phase estimation is observed and analyzed. The performance analysis of generalized extended state observer (ESO) in handling fast-varying sinusoidal disturbances is presented in Literature [12].Most of the literature studies the accuracy and rapidity of the estimation of the unknown disturbances and states. The ESO often uses high gain to 
ensure the estimation accuracy. When a big error is existed in initial value between ESO and the observed system status, derivative peaking phenomena is appeared generally in ESO. ${ }^{[3]}$

This paper gives the general form of ESO with hyperbolic sine nonlinear function based on the analysis of the hyperbolic sine function characteristic. A Lyapunov function is used to prove the asymptotically stable of second order extended state observer error system. And variable parameters ESO is designed with saturated characteristics of hyperbolic tangent function to suppress derivative peaking phenomena

\section{The design of extended state observer based on hyperbolic sine function}

\subsection{The characteristics of hyperbolic sine function}

The hyperbolic sine function is a singular function, which is monotonically increasing and smooth continuous in $(-\infty,+\infty)$. The derivative of the hyperbolic sine function is $\cosh (x)$, which never equal to 0 . The range of a hyperbolic sine function is $(-\infty,+\infty)$; when $x=0, \sinh (x)=\sinh (0)=0$; Hyperbolic sine function satisfies the selection conditions of nonlinear functions in the nonlinear ESO by literature [2,13].

The hyperbolic sine nonlinear function is defined as: $\operatorname{sinhFal}(e, \alpha, \beta)=\alpha \cdot \sinh (\beta \cdot e)$. In the equation, $\mathrm{e}$ is function input variable, $\alpha, \beta$ are variables which can adjust the function value and the change rate of sinhFal function. The hyperbolic sine nonlinear function is compared with the conventional Fal function, and the function values are represented by $\mathrm{y}$, as shown in Figure 1. In Figure 1, in the neighborhood of $x=0$, when $|x|$ is small, that is to $\operatorname{say} x \rightarrow 0, \sinh (x) \rightarrow x$, the hyperbolic sine function approximates the linear function, and the slope is small, in error range $|e| \leq \delta$ ( $\delta>0$,adjustable parameters) can take into account the effect of filtering and observation.

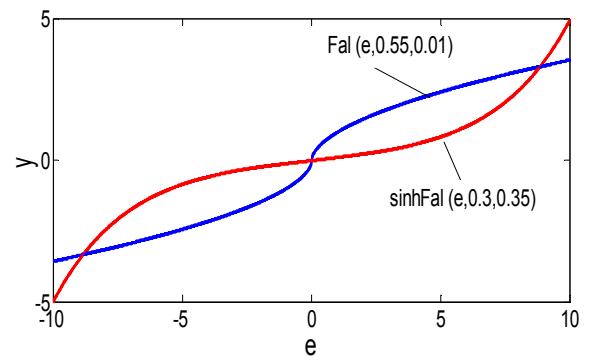

Fig.1 Comparison of hyperbolic sine nonlinear function and classical Fal nonlinear function

\subsection{The design of extended state observer based on hyperbolic sine function}

For the system:

$$
\dot{x}_{1}=f\left(x_{1}, x_{2}, \mathrm{v}(\mathrm{t})\right)+b u(t)
$$

In the equation, $b>0, f(\cdot)$ is a function of the system containing unknown nonlinear disturbances, and it is bounded. $v(t)$ is unknown disturbances. $u(t)$ is the system control input.

Make $x_{2}=f(\cdot)$ as expansion state variable of the system, and express it as $\dot{x}_{2}=\omega(t)$. Make $y(t)$ as the output for the system. Then the system can be expanded to:

$$
\left\{\begin{array}{l}
\dot{x}_{1}=x_{2}+b u(t) \\
\dot{x}_{2}=\omega(t) \\
\mathrm{y}(\mathrm{t})=x_{1}
\end{array}\right.
$$

The ESO for the above nonlinear system can be constructed with the nonlinear hyperbolic sine function: 


$$
\left\{\begin{array}{l}
e_{1}(\mathrm{t})=z_{1}(\mathrm{t})-y(\mathrm{t}) \\
\dot{z}_{1}(\mathrm{t})=z_{2}(\mathrm{t})-a_{1} \cdot e_{1}(t)+\mathrm{b} \cdot \mathrm{u}(\mathrm{t}) \\
\dot{z}_{2}(\mathrm{t})=-\operatorname{sinhFal}\left(e_{1}(t), a_{2}, b_{2}\right)
\end{array}\right.
$$

In the equation: $a_{1}>0 、 a_{2}>0 、 b_{2}>0$,selecting parameters $a_{1} 、 a_{2}$ appropriately, therefore the ESO (3) can accurately estimate all the state variables of the system : ${ }_{1}(t) 、 x_{2}(t)$.That is $z_{1}(t) \rightarrow x_{1}(t) 、 z_{2}(t) \rightarrow x_{2}(t)$, and $x_{2}(t)=f(\cdot)$

\subsection{Proof of stability of the error system for the ESO}

$$
\begin{aligned}
e_{1}(t) & =z_{1}(t)-y(t)=z_{1}(t)-x_{1}(t) \\
\text { If: } \quad e_{2}(t) & =z_{2}(t)-x_{2}(t)
\end{aligned}
$$

Assumed that $\dot{x}_{2}=\omega(t)$ and $|\omega(\mathrm{t})| \leq \omega_{0}, \omega_{0}$ is a constant and $\omega_{0} \geq 0$. The error equations of ESO system (3) and ESO system (2) are:

$$
\left\{\begin{array}{l}
\dot{e}_{1}(t)=e_{2}(t)-a_{1} e_{1}(t) \\
\dot{e}_{2}(t)=-\operatorname{sinhFal}\left(e_{1}(t), a_{2}, b_{2}\right)-\omega_{0}
\end{array}\right.
$$

The asymptotically stable of ESO error system (4) at the equilibrium point is proved as follows:

Mark: $X_{1}=e_{1}(t), X_{2}=e_{2}(t)-a_{1} e_{1}(t)$, the system (4) can be expressed as:

$$
\left\{\begin{array}{l}
\dot{X}_{1}=X_{2} \\
\dot{X}_{2}=-\sinh \operatorname{Fal}\left(e_{1}(t), a_{2}, b_{2}\right)-\omega_{0}-a_{1} X_{2}
\end{array}\right.
$$

Constructing the Lyapunov function for system (5).In the neighborhood of $e_{1}(t)=0$, $\sinh \left(b_{2} \cdot e_{1}(t)\right) \approx b_{2} \cdot e_{1}(t)$, so the nonlinear function of the formula (5) can be substituted for $b_{2} \cdot e_{1}(t)$, making it as a linear system, the quadratic function is constructed as follows:

$$
W(t)=-a_{2} \cdot b_{2} \cdot X_{2}^{2}
$$

Lyapunov function is gained as follows by using Barr Bhasin formula[14] :

$$
\begin{gathered}
V(t)=\frac{\left|\begin{array}{cccc}
0 & X_{1}^{2} & 2 X_{1} X_{2} & X_{2}^{2} \\
0 & 1 & -a_{2} b_{2} & 0 \\
0 & 0 & -a_{1} & -a_{2} b_{2} \\
-a_{1} & 0 & 1 & -a_{1}
\end{array}\right|}{\left|\begin{array}{ccc}
0 & -a_{2} b_{2} & 0 \\
1 & -a_{1} & -a_{2} b_{2} \\
0 & 1 & -a_{1}
\end{array}\right|} \\
=a_{2} b_{2} X_{1}^{2}+X_{2}^{2}
\end{gathered}
$$

Lyapunov function which can restore nonlinear system is expressed as :

$$
V(t)=2 a_{2} \int_{0}^{e_{1}(t)} \sinh \left(b_{2} \cdot e_{1}(t)\right) d e_{1}(t)+X_{2}^{2}
$$

The derivation of it is:

$$
\begin{aligned}
& \dot{V}(t)=\frac{\partial V}{\partial X_{1}} \dot{X}_{1}+\frac{\partial V}{\partial X_{2}} \dot{X}_{2} \\
& =2 a_{2} \sinh \left(b_{2} e_{1}(t)\right) \cdot X_{2}+2 X_{2}\left(-a_{2} \sinh \left(b_{2} e_{1}(t)\right)-\omega_{0}-a_{1} X_{2}\right) \\
& =-2 X_{2}\left(\omega_{0}+a_{1} X_{2}\right)
\end{aligned}
$$


Therefore, when the perturbation $\omega_{0}=0$, because of $a_{1}>0$ and $X_{2}$ is not always equal to 0 ,so $\dot{V}(t)=-2 a_{1} X_{2}^{2}<0$. The Lyapunov function which is constructed according to the system (5) is effective, and the zero solution of the system (5) is Lyapunov asymptotic stability.

When the disturbance $\omega_{0} \neq 0$, as long as $X_{2}\left(\omega_{0}+a_{1} X_{2}\right)>0$, there is $\dot{V}(t)<0$.Considering the amount of interference $|\omega(\mathrm{t})| \leq \omega_{0}$ and $\omega_{0} \geq 0$, when the system (5) is at the steady state, there is:

$$
\left\{\begin{array}{l}
\dot{X}_{1}=X_{2}=0 \\
\dot{X}_{2}=-a_{2} \sinh \left(b_{2} \cdot e_{1}(t)\right)-\omega_{0}-a_{1} X_{2}=0
\end{array}\right.
$$

Take the absolute values for second equation: $\left|\sinh \left(b_{2} \cdot e_{1}(t)\right)\right|=\frac{\omega_{0}}{a_{2}}$.. As the hyperbolic sine function is monotone increasing function, the observation error of state observer can beexpressed respectively as:

$$
\begin{aligned}
& \left|e_{1}(t)\right| \leq \frac{1}{b_{2}} \sinh ^{-1}\left(\frac{\omega_{0}}{a_{2}}\right) \\
& \left|e_{2}(t)\right| \leq \frac{a_{1}}{b_{2}} \sinh ^{-1}\left(\frac{\omega_{0}}{a_{2}}\right)
\end{aligned}
$$

\section{Design of Time-varying parameter based on hyperbolic tangent function}

When there is a big error in the initial value between the ESO state and system state variable, in the vicinity of the initial time of ESO, there will be a larger peak existing in the derivative signal estimation and acceleration signal estimation of the system. When the gain coefficient is relatively large, it will enable ESO in greater derivative peak.

In order to eliminate derivative peak of the ESO, the ESO parameter values $a_{1} 、 a_{2}$ in the equation(3) is set by using the saturation characteristics of hyperbolic tangent function, variable parameters $a_{11}$ 、

$a_{22}$ is used to replace it. The parameter values $a_{11} 、 a_{22}$ have time-varying characteristics, they are as follows:

$$
\left\{\begin{array}{l}
a_{11}=a_{1} \cdot \tanh \left(b_{1} \cdot t\right) \\
a_{22}=a_{2} \cdot \tanh \left(b_{2} \cdot t\right)
\end{array}\right.
$$

In the equation (13), $a_{1} 、 a_{2}$ are the adjustable parameters of ESO in specific observation system when observing specific system. Due to the robustness of the ESO, the adjustable parameters have a large range, which can be designed according to Ref. [10] "dynamic parameters determining method". $b_{1} 、 b_{2}$ are coefficient of the time-varying parameter, they are adjustable parameters. Because of the characteristics of hyperbolic tangent function, the value $a_{11} 、 a_{22}$ will be relatively small in the initial stage of ESO operation. By choosing the appropriate time-varying parameter coefficient, the characteristic will restrain the derivative peak in the initial time of ESO .

\section{Simulation analysis of time-varying parameters ESO based on hyperbolic sine function}

Simulation and verification of two order time-varying parameters ESO based on hyperbolic sine function will be given as follows. ESO based on hyperbolic tangent function is designed through time-varying parameters in formula(13),inspecting its estimation effect, and then comparing with estimation effect of ESO based on classical Fal function in literature [2]. 
Assuming a first order system model as:

$$
\left\{\begin{array}{l}
\dot{x}_{1}=2 \cdot \sin (\cos (0.5 t))+b u(t) \\
\dot{x}_{2}=\omega(t) \\
y(t)=x_{1}
\end{array}\right.
$$

Assuming that the nonlinear sinusoidal disturbances $f(t)=2.0 \sin (\cos (0.5 t))$ is unknown. The input is $u(t)=\cos (0.5 t)$, and $b=3$. Use the formula (3) to construct the second order ESO as follows:

$$
\left\{\begin{array}{l}
e_{1}(t)=z_{1}(t)-y(t) \\
\dot{z}_{1}(t)=z_{2}(t)-a_{11} b_{1} \cdot e_{1}(t)+b \cdot u(t) \\
\dot{z}_{2}(t)=-a_{22} \cdot \alpha \sinh \left(\beta \cdot e_{1}(t)\right)
\end{array}\right.
$$

Parameter values of the ESO are designed according to literature[13] "dynamic parameters determining method". The poles of the compensation matrix are $p_{1,2}=-10$, and $a_{1}=20$, $a_{2}=75, b_{1}=1, b_{2}=1.33, \alpha=3, \beta=0.3$; The initial state of the system ${ }^{x_{1}}$ is 2 , and the initial value of ESO is $[0,0] . a_{11} 、 a_{22}$ use the time-varying parameters of the formula (11). The value of $a_{11} 、 a_{22}$ in the time $0<t<15$ is shown in Figure 2.The time-varying parameters have a smaller value at the initial moment, which can realize the suppression of derivative peak. Due to the saturation characteristic of hyperbolic tangent function, in the behind times, the parameters can be reached ESO tuning parameter values, which can guarantee the estimation accuracy of the ESO.

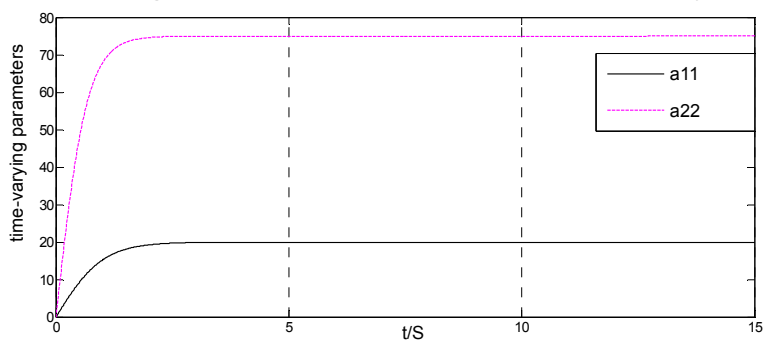

Fig.2 Value of time-varying parameters $a_{11}$ and $a_{22}$

The system state $x_{2}(t)$ and the estimated values in different nonlinear functions are shown in Figure 3.Estimated error value on system state ${ }^{X_{2}}$ of ESO with different nonlinear function are shown in Figure 4. As it can be seen from Figure 3, in the initial stage, when the error between initial value of the ESO state and the initial value of system state variable is 2, the estimation of ESO based on classical Fal nonlinear function in literature [2] to the state of $x_{2}(t)$ which is the derivative state of system state $X_{1}(t)$ appeared the peak phenomenon. The maximum peak of the estimation of the nonlinear perturbation signal $f(t)$ is about 9.45 , which is 4.725 times the error of its initial value. If the peak signal is used to determine the input of the system, the control effect will be affected.Time-varying parameters ESO based on the sinhFal nonlinear function can restrain the peak estimation of the state ${ }^{X_{2}(t)}$.From Figure 4, in the initial stage, the estimation error of ESO based on Fal nonlinear function to the state of the system $x_{2}(t)$ is about 7.76, and the estimation of the time-varying parameters ESO based on the sinhFal nonlinear function is within the range of its intrinsic error, the maximum is 1.59 , which is $1 / 4.88$ times the estimation error of the ESO based on Fal nonlinear function. 


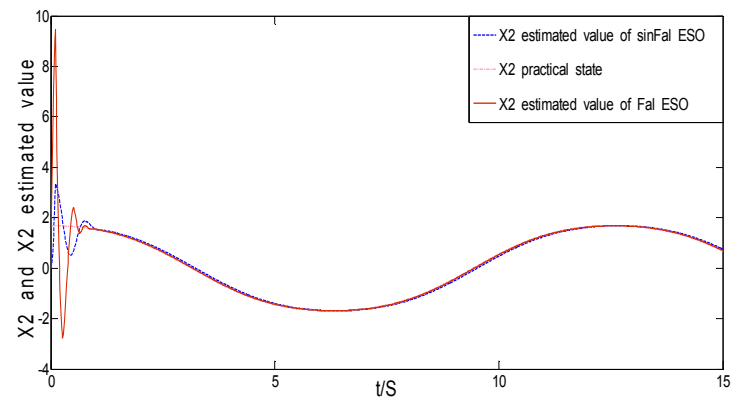

Fig.3 System state $x_{2}$ and estimated value of ESO with different nonlinear function

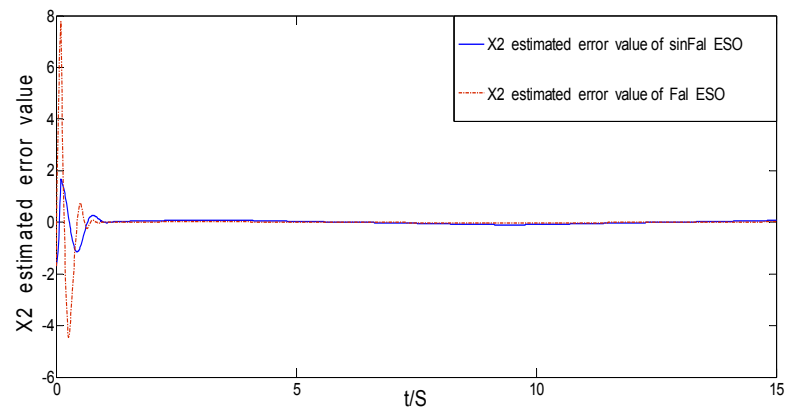

Fig.4 Estimatederror value on system state $x_{2}$ of ESO with different nonlinear function

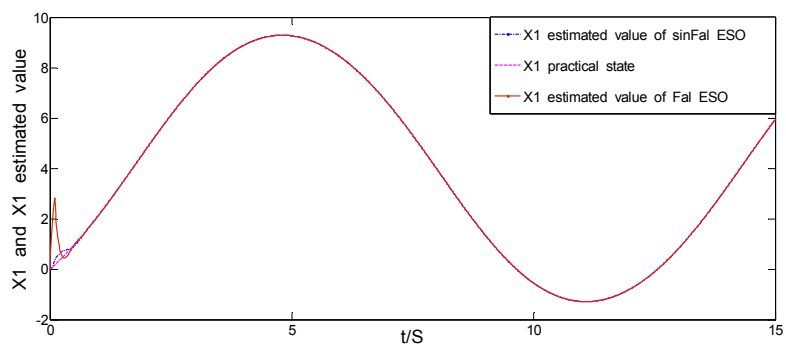

Fig.5 System state $x_{1}$ and estimated value of ESO with different nonlinear function

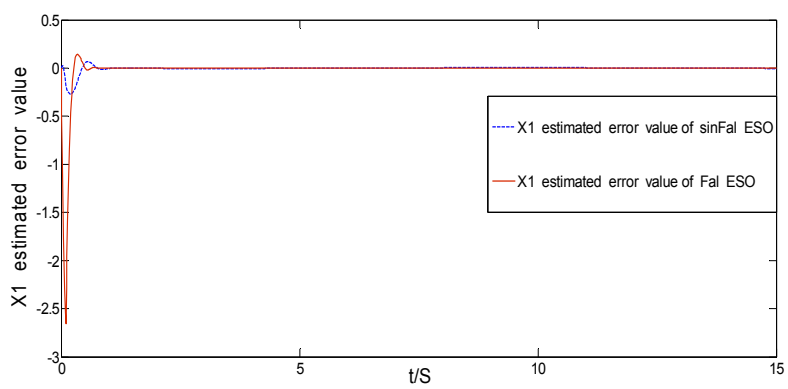

Fig.6 Estimatederror value on system state $x_{1}$ of ESO with different nonlinear function

The system state ${ }^{X_{1}}(t)$ and the estimated values in different nonlinear functions are shown in Figure 5. Estimated error value on system state ${ }^{X_{1}}$ of ESO with different nonlinear function are shown in Figure 6. It can be seen from figure 5 and figure 6 that, in the initial stage, when the error between initial value of the ESO state and the initial value of system state variable is 2, the estimation of ESO based on Fal nonlinear function to the state of the system $x_{1}(t)$ also has the derivative peak phenomenon, the maximum estimation error is 2.66.The ESO based on the nonlinear function tanhFal can effectively inhibit the estimation peak in the initial stage, and its estimation error is 0.28 . At the same time, the ESO based on the nonlinear function tanhFal can obtain higher system state variable estimation accuracy at the other stage. 


\section{Conclusion}

The hyperbolic sine function is monotonically increasing and continuous smooth nonlinear function. It has fast linear response in the neighborhood of 0 . The general form of the ESO based on the hyperbolic tangent function is presented in this paper. It is proved that the error system of second order ESO is asymptotically stable at the equilibrium point. The extended state observer (ESO) with time-varying parameters which based on the saturation characteristic of hyperbolic tangent function was designed to realize the effectiveness of derivative peaking phenomena. Simulation results show that time-varying parameters ESO based on the hyperbolic sine nonlinear function can effectively suppress the derivative peak phenomenon and it can accurately observe the state variables and nonlinear disturbances of the system. So the ESO designed in this paper has the reference value for the ESO by using high gain design to eliminate system internal and external interference and ensure the estimation accuracy of the system.

Supported by the Fundamental Research Funds for the Central Universities (14CX05039A), Supported by National Natural Science Foundation of China (61271001).

\section{References}

[1] Huang Y, Xue W C. Active disturbance rejection control: Methodology, applications and theoretical analysis[J]. Jof Systems Science and Mathematical Sciences, 2012, 32(10):1287-1307.

[2] Han J Q. Active disturbance rejection control technique-The technique for estimating and compensating the uncertainties[M]. Beijing: National Defense Industry Press, 2008:197-208.

[3] Wang X H, Liu J K. Differentiator design and application-signal filtering and differentiation[M]. Beijing: Publishing House of Electronics Industry, 2010: 144-155.

[4] Shao L W, Liao X Z, Xia Y Q, et al. Stability analysis and synthesis of third order discrete extended state observer[J]. Information and Control, 2008, 37(2): 135-139.

[5] Wang Y H, Yao Y, Ma K M. Error estimation of second order extended state observer[J]. Journal of Jilin University, 2010, 40(1): 143-147.

[6] Zhou L Y, Wang S J. An improved ADRC based-on nonlinear arctangent function[J]. Journal of Shanghai Jiaotong University, 2013, 47(7): 1043-1048.

[7] Li S Q, Zhang S X, Liu Y N, et al. Parameter-tuning in active disturbance rejection controller using time scale [J]. Control Theory \& Applications, 2012, 29(1): 125 -129.

[8] Yang M, Dong C, Wang S Y, Chao T. Linear Extended State Observer Based on Finite-time Output Feedback[J]. Acta Automatica Sinica, 2015, 41(1): 59-66.

[9] A. A. Godbole, T. R. Libin, and S. E. Talole, Extended state observer-based robust pitch autopilot design for tactical missiles[J].Proc. IMechE,Part G.2012,226(12): 1482-1501.

[10] Y. Xia, Z. Zhu, and M. Fu.Back-stepping sliding mode control for missile systems based on an extended state observer[J]. IET Control Theory Appl. 2011, 5(1):93-102.

[11] Bao-Zhu Guo, Zhi-liang Zhao. On the convergence of an extended state observer for nonlinear systems with uncertainty [J]. Systems \& Control Letters, 60 (2011): 420-430.

[12] Ashwini A. Godbole, Jaywant P. Kolhe, and Sanjay E. Talole. Performance Analysis of Generalized Extended State Observer in Tackling Sinusoidal Disturbances [J]. IEEE Transactions on Control Systems Technology, 2013, 21(6):2212-2223.

[13] Kang Z J, Chen X Y. A design method of nonlinear extension state observer[J]. Electric Machines and Control, 2001, 5(3): 199-203.

[14] Yang W L, Liang L H, Teng G L. Stability analysis for Nonlinear Systems[J]. Journal of Tianjin University, 1995, 28(6): 759-764. 